\title{
Energy Economics in the Manufacturing Industry: A Return on Investment Strategy
}

\author{
Michael P Brundage ${ }^{1 \mathrm{a}}$, Qing Chang ${ }^{\mathrm{a}}$, Jing Zou ${ }^{\mathrm{a}}$, Yang $\mathrm{Li}^{\mathrm{b}}$, Jorge Arinez ${ }^{\mathrm{c}}$, Guoxian \\ $\mathrm{Xiao}^{\mathrm{c}}$ \\ ${ }^{a}$ Dept of Mechanical Engineering, Stony Brook University Stony Brook, New York 11794 \\ ${ }^{b}$ Department of Mechanical Engineering, University of Michigan, Ann Arbor, MI 48105 \\ ${ }^{c}$ General Motors RESD, General Motors Corporation, Warren, MI 48090
}

\begin{abstract}
Manufacturers lack the economic tools to properly perform maintenance procedures to increase manufacturing reliability while reducing system energy consumption. A return on investment strategy is developed to provide plant managers with a quantitative method to optimize the maintenance actions that lead to the largest return on investment. Over the long term, this energy economic analysis results in the largest decrease in energy costs for a manufacturing facility. A control methodology is developed to increase profits on a daily basis by inserting energy opportunity windows at various machines thus reducing energy consumption with minimal production impact. A simulation case study is performed to validate the return on investment strategy and to test the control methodology's impact on the overall profit of the facility.
\end{abstract}

Keywords: Energy Economics, Return on Investment, Energy Control Methodology, Energy Profit Optimization, Continuous Process Improvement, Preventative Maintenance Strategies

\section{INTRODUCTION}

In 2013, the energy consumption in the United States within the industrial sector was 31.15 quadrillion BTU; by 2020 it is projected to reach 35.76 quadrillion BTU $[1,2]$. Energy consumption in industry is projected to grow at an annual rate of $0.8 \%$ per year, which is the largest projected increase among any sector within the country. From 2006 - 2010, the average energy price for electricity usage within the

\footnotetext{
${ }^{1}$ Michael.Brundage@stonybrook.edu, ph: 631-632-8329, fax: 631-632-8544

Preprint submitted to Energy

September 16, 2015
} 
manufacturing sector has increased 3\% [3]. It is imperative for manufacturers to reduce energy consumption without affecting production, thus reducing costs within the facility.

Previous energy management research within the manufacturing industry mainly focused on maintaining throughput or quality control without explicitly considering energy consumption $[4,5]$. These studies treat energy consumption as a byproduct of production instead of treating it as the main driver in the decision making process. However, in [6] it is discovered that there is a positive effect of energy and environmental improvement investment on overall production growth. To achieve sustainability within the manufacturing sector, it is necessary to focus on reducing the overall energy demand of the facility, not just maintaining throughput targets.

Maintenance prioritization is a research area dedicated to improving system performance through the scheduling of reactive and preventative maintenance [7]. Most maintenance policies focus on heuristic rules or expert knowledge to determine when and at which machine to perform maintenance [8]. These policies focus on reactive maintenance and are limited by the knowledge of the plant manager at the facility. Yang et al. discuss prioritization strategies by using online production data to optimize maintenance policy [9]. However, machine replacement strategy is not discussed in this work. In $[10,11]$, preventative maintenance strategies are discussed by managing availability of maintenance staff and production requirements. This paper focuses on preventative maintenance and the energy economics of replacing a part or entire machine on the production line.

Recent studies on manufacturing efficiency have focused on the creation of sustainable indicators, but these vary greatly from industry to industry. In [12], the authors review various sustainability indicators and manufacturing processes, but methodologies on how to reduce the facility energy consumption are not presented. A fuzzy-based sustainable manufacturing assessment model is created for sustainability of medium and small enterprises in [13], however this only applies to a small number of sustainability indicators and cannot be expanded for more general systems. Sustainable manufacturing performance indicators are created in [14] that analyze the dynamic energy structure of the production system. However, this work does not explicitly address the production system economic impact. Despeisse et al. study the strategic practices of manufacturing facilities to extract the mechanisms behind the processes to formulate sustainable manufacturing tactics [15]. This method relies on simulation modeling to guide the plant manager in decision making, but does not explicitly consider economic impacts on the production floor.

There exists a gap between energy efficiency performance measurement introduced in scientific literature and what is used within industry [16]. Bunse et al. 
interviewed over 100 industry experts who stated that the measurement of energy efficiency needed to be converted to monetary values to communicate directly where there would be monetary savings. Another problem with implementing energy efficiency improvement is the difficulty of measuring energy indicators within the manufacturing industry [17]. The method developed in this paper provides the plant manager with the quantitative tools that utilize readily available sensor data to directly predict the energy cost savings of performing maintenance on specific machines on the manufacturing line. A control methodology that uses real sensor data to selectively turn off certain machines to reduce the overall energy cost with minimal impact to production is also introduced.

Demand side management focuses on the reduction of the energy demand of the manufacturing plant. Two methods are typically utilized: electricity demand response and energy efficiency improvement [18]. Electricity demand response shifts patterns of electricity usage therefore reducing the demand on the electrical grid. This idea is explored in [19] where the facility heating, ventilation, and air conditioning (HVAC) system is integrated with the production operations to optimize energy cost savings.

The contribution of this paper is to analyze the energy consumption of dynamic manufacturing systems and to improve energy efficiency through an integrated control scheme. The energy related cost and energy economics are explicitly considered in the control objective function to trade off various machine repair and replacement decisions. Energy efficiency improvement reduces energy consumption with minimal throughput loss, which is the main concern of this paper.

The remainder of the paper is structured as follows: Section 2 provides the assumptions and background. In Section 3, the authors present an energy economic analysis. Section 4 provides a control methodology and Section 5 includes numerical case studies. Finally, we discuss the results and provide future work in Section 5.

\section{Nomenclature}

- $b_{m}(t), m=2,3, \ldots, M$, represents the buffer level of buffer $B_{m}$ at time $t$.

- $\vec{e}_{j, i}=\left(j, t_{i}, d_{i}\right), i=1,2 \ldots, \eta_{j}, j=1,2, \ldots, M$, is the $i^{t h}$ downtime event for machine $j$, which occurs at time $t_{i}$ for a duration of $d_{i}$ time units.

- $\vec{e}_{j}=\left\{\vec{e}_{j, 1}, \vec{e}_{j, 2}, \ldots, \vec{e}_{j, \eta_{j}}\right\} j=1,2, \ldots, M$, denotes a sequence of downtime events for machine $j$ where $\eta_{j}$ is the number of downtime events at machine $j$.

- $\vec{E}=\left\{\vec{e}_{1}, \vec{e}_{2}, \ldots, \vec{e}_{M}\right\}$ represents a sequence of downtime events for the line. 
- $\frac{1}{\tau_{j}}, j=1,2, \ldots, M$ denotes the rated speed of machine $j$.

- $s_{j}(t), j=1,2, \ldots, M$ represents the actual processing speed of machine $j$ at time $t$.

- $\int_{0}^{t} s_{j}\left(t^{\prime}, \vec{E}\right) d t^{\prime}, j=1,2, \ldots M$ is the production volume of machine $j$ from $[0, \mathrm{t})$ subject to a set of downtime events $\vec{E}$.

- $C T R_{j}^{k}$ is the cost to replace a part $k$ within machine $j$. The scenario where the entire machine $j$ is replaced is represented by $k=0$.

- $c_{p}$ denotes the profit per part produced in $\$ /$ part.

- $c_{e}$ represents the cost of electrical energy in $\$ / k W h$.

- $c_{d}$ is the demand cost if the production count does not meet demand.

- $c_{b}$ represents the back log cost if the production count is below demand.

- $c_{i}$ is the inventory cost if the production count is above demand.

- $c_{r e p, j}^{k}$ is cost to replace part $k$ at machine $j$.

- $N_{m, j}^{k}$ represents the number of maintenance workers required to replace part $k$ in machine $j$.

- $t_{R, j}^{k}$ is the repair time required to replace part $k$ in machine $j$.

- $c_{\text {Main }, j}$ is the cost per hour of a maintenance worker at machine $j$.

- $C_{R e g, j}$ is the cost per hour of a maintenance worker at machine $j$ if the maintenance is performed during a regular shift.

- $C_{O T, j}$ is the cost per hour of a maintenance worker at machine $j$ if the maintenance is performed during an overtime shift.

- $R O I_{j}^{k}$ denotes the expected return on investment for replacing part $k$ within machine $j$ in $[0, T)$.

- $B E_{j}^{k}$ represents the expected number of days for an investment on part $k$ at machine $j$ to break even.

- $\eta_{O W, j}$ is the number of inserted opportunity windows at machine $j$.

- $t_{O W, j}$ denotes the length of one inserted opportunity window at machine $j$. 


\section{ASSUMPTIONS AND BACKGROUND}

This paper adopts a continuous flow model [20, 21, 22]. The continuous flow model treats the quantity of jobs in a buffer as varying continuously from zero to the capacity of the buffer, as opposed to varying in integer steps. This is done for ease of analysis since the system dynamics can be conveniently expressed as integral or differential equations. The system dynamics are not affected by this assumption regardless of whether the system is discrete or continuous [22, 23, 24]. A serial production line with with $\mathrm{M}$ machines (represented by the rectangles) and M-1 buffers (represented by the circles) is shown in Fig. 1. For the serial production

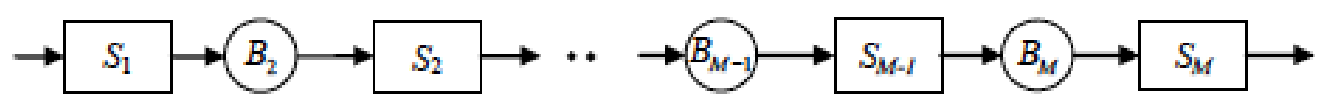

Figure 1: A SERIAL PRODUCTION LINE WITH M MACHINES AND M-1 BUFFERS

line as represented in Fig. 1, we make the following assumptions:

1. Each machine $j$ has a rated speed $\frac{1}{\tau_{j}}, j=1,2, \ldots, M$, where $\tau_{j}$ is the cycle time of the machine. An operational machine can process jobs at a speed that is no greater than its rated speed. The machine can be viewed as operating at a duty cycle less than one when its speed is constrained by other machines, i.e. a machine is starved/blocked by another machine. We denote $s_{j}(t), j=$ $1,2, . ., M$, as the actual processing speed of machine $j$ at time $t$. The production volume of machine $j$ from $[0, \mathrm{t})$ is represented by $\int_{0}^{t} s_{j}\left(t^{\prime}\right) d t^{\prime}, j=1,2, \ldots M$.

2. $M^{*}=\operatorname{argmin}_{j=1, \ldots M}, \frac{1}{\tau_{j}}$ represents the slowest machine in the line and it is unique.

3. Each buffer $B_{2}, B_{3}, \ldots, B_{M}$ has a finite capacity. $B_{2}, B_{3}, \ldots, B_{M}$ also denotes the maximum capacity of the buffer.

4. A machine is blocked if it is operational and its downstream buffer is full.

5. A machine is starved if it is operational and its upstream buffer is empty.

6. The first machine, $j=1$, is never starved and the last machine, $j=M$, is never blocked.

7. A machine cannot breakdown if it is blocked or starved.

8. The power consumption of each machine is zero when the machine is completely turned off, during $T_{o f f, j}$.

9. Machine $j$ has a warmup time of $t_{w, j}^{i}$ for the $i^{t h}$ downtime event, where $t_{w, j}^{i} \sim$ $\exp \left(\omega_{j}\right)$ and $\omega_{j}$ is the mean time machine $j$ warms up. 
It is necessary to understand how random downtime events affect the system when analyzing the energy dynamics of the production line. The energy opportunity window, $W_{j}\left(T_{d}\right)$, is the amount of time machine $j$ can be down at time $T_{d}$ without resulting in permanent production loss [25, 26, 27]. The largest possible downtime event at machine $j$ without resulting in permanent production loss due to the $i^{\text {th }}$ failure event, $\vec{e}_{j, i}=\left(j, t_{i}, d_{i}\right)$ is $d_{i}^{*}$ :

$$
d_{i}^{*}=\inf \left\{d \geq 0: \text { s.t. } \tau_{M^{*}} \int_{t_{i}}^{t_{i}+d} s_{M^{*}}\left(t^{\prime} ; \vec{E}\right) \mathrm{d} t^{\prime}=W_{j}\left(t_{i}\right)\right\},
$$

where $d_{i}^{*}$ is the time it takes the buffers between machine $j$ and $M^{*}$ to become full if $j>M^{*}$ or empty if $j<M^{*}$. $W_{j}\left(t_{i}\right)$ is the energy opportunity window at time $t_{i}$ for machine $j$, which represents the longest time that machine $j$ can be turned down for energy savings without leading to production throughput loss as seen at a certain time $T>t_{i}+d$. If a downtime event is greater than the value presented in Eq. 1 then there is permanent production time loss of $d_{i}-d_{i}^{*}$. This is referred to as an effective downtime event [25]. A non-effective downtime event is when the duration is less than the above value $\left(d_{i} \leq d_{i}^{*}\right)$, and thus will not lead to permanent production loss. However, this is under the assumption that the warm up time $\left(t_{w, j}^{i}\right)$ of machine $j$ is equal to zero for the $i^{t h}$ downtime event. Since a machine is consuming energy without producing parts during this warm up time period, it must be taken into account when analyzing the production system energy consumption. The period that machine $j$ does not produce parts is $d_{i}+t_{w, j}^{i}$. Let $\vec{D}^{e}$ represent a set of $\eta_{j}^{e}$ number of effective downtime events and $\vec{D}^{n}$ represent a set of $\eta_{j}^{n}$ number of non-effective downtime events, then $\forall d_{i}$ :

$$
d_{i} \in \begin{cases}\vec{D}^{n}, & d_{i} \leq\left(d_{i}^{*}-t_{w, j}^{i}\right), \quad i=1 \ldots \eta_{j} \\ \vec{D}^{e}, & d_{i}>\left(d_{i}^{*}-t_{w, j}^{i}\right), \quad i=1 \ldots \eta_{j}\end{cases}
$$

where:

$$
\eta_{j}=\eta_{j}^{e}+\eta_{j}^{n}
$$

Thus, an effective downtime event leads to permanent production time loss of $d_{i}-$ $d_{i}^{*}+t_{w, j}^{i}$. 


\section{ENERGY ECONOMIC ANALYSIS}

It is imperative to study the energy economics of the manufacturing system to reduce overall energy costs. First, we analyze the profit in the time period $[0, T)$ :

$$
\begin{aligned}
\text { Profit } & =\text { Income }- \text { Expenses } \\
& =P C\left(c_{p}\right)-E\left(c_{e}\right)-|P C-D|\left(c_{d}\right)-C T R_{j}^{k},
\end{aligned}
$$

where $P C$ is the production count, which is measured by sensor data. The energy consumption of the production line is represented by $E$. The production demand of the plant in the time period $[0, \mathrm{~T})$ is $D$ and $c_{e}$ and $c_{p}$ are cost of energy per $k W h$ and the profit per part respectively. We will assume that the expenses to produce the part in terms of material and labor are already considered in the profit per part. The cost to replace, $C T R_{j}^{k}$, is included if machine $j$ is replaced with a more energy efficient machine or a part $k$ is replaced within machine $j$ to reduce energy consumption. The third term represents the cost incurred if production is less than demand $(P C<D)$, leading to a back log cost or if production is greater than demand $(P C>D)$, leading to an inventory cost. The demand cost, $c_{d}$, is calculated by:

$$
c_{d}= \begin{cases}c_{b}, & P C<D \\ 0, & P C=D \\ c_{i}, & P C>D\end{cases}
$$

where $c_{i}$ is the cost of inventory per part and $c_{b}$ is the back log cost per part. To find the energy consumption, $E$, we analyze how each machine operates in $[0, T)[28]$ :

$$
T=T_{p p, j}+T_{w, j}+T_{i d, j}+T_{o f f, j} .
$$

The energy consumption of the production line in $[0, \mathrm{~T})$ is represented by:

$$
E=\sum_{j=1}^{M} T_{p p, j} P_{p p, j}+T_{i d, j} P_{i d, j}+T_{w, j} P_{w, j}+T_{o f f, j} P_{o f f, j},
$$

where $T_{p p, j}$ is the time machine $j$ is producing parts with a power consumption of $P_{p p, j}$ and the idle time of machine $j$ is $T_{i d, j}$ with a power consumption of $P_{i d, j}$. The warmup time of machine $j$ is $T_{w, j}$ consuming power at a rate of $P_{w, j}$, and the time machine $j$ is off is $T_{o f f, j}$ while it consumes power at a rate of $P_{o f f, j}$. The power consumed while the machine is off is zero per our assumption. We can assume that the power consumed while the machine is idling is a percentage of the power consumed during the production of parts:

$$
P_{i d, j}=\alpha_{j} P_{p p, j}, \quad 0 \leq \alpha_{j} \leq 1 .
$$


The same assumption can be made for the power consumed during machine warm up, however the power consumed while the machine is warming up is not always less than the power consumed during the production of parts. These values can be calculated based on ratings given by the machine manufacturers.

$$
P_{w, j}=\beta_{j} P_{w, j}, \quad \beta_{j} \geq 0 .
$$

Thus, Eq. 4 becomes:

$$
E=\sum_{j=1}^{M} P_{p p, j}\left(T_{p p, j}+\beta_{j} T_{w, j}+\alpha_{j} T_{i d, j}\right) .
$$

The time that machine $j$ produces parts is:

$$
T_{p p, j}=\tau_{j} \int_{0}^{T} s_{j}\left(t^{\prime} ; \vec{E}\right) \mathrm{d} t^{\prime}
$$

and the warm up time is represented by:

$$
T_{w, j}=\sum_{k=1}^{\eta_{j}} t_{w, j}^{k}
$$

where $\eta_{j}$ is the number of downtime events at machine $j$ and $t_{w, j}^{k}$ is the length of time machine $j$ warms up after the $k^{\text {th }}$ breakdown. The time that machine $j$ is off is:

$$
T_{o f f, j}=\sum_{k=1}^{\eta_{j}} d_{k} .
$$

The time that machine $j$ is idle due to being blocked/starved is displayed in Eq. 11:

$$
T_{i d, j}=T-\tau_{j} \int_{0}^{T} s_{j}\left(t^{\prime} ; \vec{E}\right) \mathrm{d} t^{\prime}-\sum_{k=1}^{\eta_{j}}\left[d_{k}+t_{w, j}^{k}\right] .
$$

Plugging into Eq. 7 and manipulating provides the energy consumption:

$$
E=\sum_{j=1}^{M} P_{p p, j}\left[T-\sum_{k=1}^{\eta_{j}}\left[d_{k}-\left(1+\beta_{j}\right) t_{w, j}^{k}\right]-\left(1-\alpha_{j}\right) T_{i d, j}\right] .
$$

It is important to note that every term in Eq. 12 can be calculated using readily available sensor data. We utilize this analysis to study the energy economics of the system. 


\subsection{RETURN ON INVESTMENT ANALYSIS}

To improve energy efficiency in the long term, it is sometimes necessary to upgrade certain parts or even entire machines to more energy efficient versions. Currently, plant managers lack the proper quantitative tools to select which part or machine should be replaced with a more energy efficient version. When replacing a part within a machine it is imperative that the investment leads to the most energy cost savings. Therefore, we utilize energy economic analysis to determine the part or machine which, when replaced, leads to the largest return on investment. Return on investment (ROI) is used in financial analysis to compare the efficiency of different investments. The investment with the highest return on investment is the one selected. The ROI is the benefit of an investment (return) divided by the cost of the investment [29]. The formula for ROI is displayed in Eq. 13:

$$
R O I=\frac{(\text { Gain from investment }- \text { Cost of investment })}{\text { Cost of investment }} .
$$

When replacing a part or machine, the cost of investment is the cost to replace the machine $j$ or a part $k$ within machine $j\left(C T R_{j}^{k}\right)$. We will denote the case $k=0$ as the scenario when the entire machine is being replaced. The cost to replace is represented by:

$$
C T R_{j}^{k}=\text { Part Cost }+ \text { Maintenance Cost }+ \text { PPL Cost }
$$

where the Part Cost is the cost of the part or machine that is being replaced: Part Cost $=c_{r e p, j}^{k}$. The Maintenance Cost is represented by:

$$
\text { Maintenance Cost }=N_{m, j}^{k} t_{R, j}^{k} c_{M a i n, j},
$$

where $N_{m, j}^{k}$ is the number of maintenance workers required at machine $j$ to replace

part $k, t_{R, j}^{k}$ is the time it takes to replace part $k$ at machine $j$ in hours, and $c_{\text {Main,j }}$ is the cost per hour for each maintenance person. The maintenance cost per person per hour depends if the maintenance is performed during a regular shift $\left(C_{R e g, j}\right)$ or within an overtime shift $\left(C_{O T, j}\right)$ :

$$
c_{\text {Main }, j}=\left\{\begin{array}{l}
C_{R e g, j}, \\
C_{O T, j}, \quad \text { during regular shift },
\end{array}\right.
$$

The last term in Eq. (14) is the permanent production loss cost. If the maintenance repair time $\left(t_{R, j}^{k}\right)$ is longer than the opportunity window when the repair is being performed then there is production loss. If the maintenance is performed within the 
opportunity window duration then there is no cost due to permanent production loss. Therefore, the permanent production loss cost is represented by:

$$
\text { PPL Cost }= \begin{cases}c_{p}\left(t_{R, j}^{k}-d_{i}^{*}\right) \frac{1}{\tau_{M^{*}}}, & t_{R, j}^{k}>d_{i}^{*} \\ 0, & t_{R, j}^{k} \leq d_{i}^{*}\end{cases}
$$

The gain from the investment is the energy cost savings from replacing a part or the entire machine, which reduces the power consumption of machine $j$ by an amount $\Delta P_{p p, j}$. Therefore, the energy savings, $\Delta E_{j}^{k}$, in the time period $[0, \mathrm{~T})$ is:

$$
\Delta E_{j}^{k}=\Delta P_{p p, j}\left[T-\sum_{k=1}^{\eta_{j}}\left[d_{k}-\left(1+\beta_{j}\right) t_{w, j}^{k}\right]-\left(1-\alpha_{j}\right) T_{i d, j}\right] .
$$

Thus, the gain from investment is calculated in Eq. 17:

$$
\text { Gain from investment }=c_{e} \Delta E_{j}^{k} \text {, }
$$

and the return on investment in the time period $[0, \mathrm{~T})$ for replacing machine $j$ or part $k$ within machine $j$ is:

$$
R O I_{j}^{k}=\frac{\left(c_{e} \Delta E_{j}^{k}-C T R_{j}^{k}\right)}{C T R_{j}^{k}} .
$$

Therefore, replacing a part within machine $j$ or replacing the entire machine with a more energy efficient version (reducing $P_{p p, j}$ by an amount $\Delta P_{p p, j}$ ) will lead to the largest return on investment in $[0, \mathrm{~T})$ if:

$$
R O I_{j}^{k}>R O I_{z}^{k}, \quad \forall z \neq j .
$$

This analysis can be applied to any time period $[0, T)$. By utilizing the ROI, the manager can select the machine or part with the largest return on investment for any time period. Another important identifier in economic analysis is the break-even point, which is the point at which the cost and the revenue are equal. This term uses return on investment to study how quickly the investment leads to a net gain in profit. In this scenario, this is the point when the energy cost saved is equivalent to the cost to replace the part or machine. Therefore, the expected number of days it takes for the investment to break even is:

$$
B E_{j}^{k}=\frac{C T R_{j}^{k}}{c_{e} \overline{\Delta E}_{j}^{k}}
$$


where $B E_{j}^{k}$ is the expected number of days it takes for the cost to replace part $k$ within machine $j$ to equal the energy cost savings at machine $j$. The average energy savings per day is $\overline{\Delta E}_{j}^{k}$. It is important to note that the machine with the maximum $R O I_{j}^{k}$ will always have the smallest $B E_{j}^{k}$ for the time period $[0, T)$. The $B E_{j}^{k}$ gives an alternative indicator to illustrate the investment of replacing a machine or a part within the machine. These indicators provide the plant manager with the quantitative tools to select the machine or part which, when replaced, leads to the largest return on investment based on energy cost savings. While this method is used for long term energy cost savings, a method to reduce overall energy consumption on a daily basis is needed. This is addressed in the next section.

\section{CONTROL METHODOLOGY}

To maximize the profits on a daily basis, we introduce a control methodology to insert energy saving opportunity windows, which lower energy consumption with minimal throughput impact. The control scheme will also lower inventory so the production rate is closer to the demand, therefore decreasing inventory costs. We utilize the concept of the energy opportunity window introduced in Sec 2 to insert $\eta_{O W, j}$ number of opportunity windows each for a length of $t_{O W, j}$ at machine $j, j=$ $1,2, \ldots, M$. This allows for various machines to be strategically turned off without affecting the overall throughput of the line and thus reducing energy consumption. The energy consumption of the production line with the inserted downtime events is represented by:

$$
E=\sum_{j=1}^{M} P_{p p, j}\left[T-\sum_{k=1}^{\eta_{j}} d_{k}-\sum_{k=1}^{\eta_{j}+\eta_{O W, j}}\left(1+\beta_{j}\right) t_{w, j}^{k}-\eta_{O W, j} t_{O W, j}-\left(1-\alpha_{j}\right) T_{i d, j}\right] .
$$

To maximize the profit of the production facility in the period $[0, T)$, it is necessary to select the length of the inserted opportunity windows $\left(t_{O W, j}\right)$ and the number of opportunity windows $\left(\eta_{O W, j}\right)$ at each machine:

$$
\max _{\substack{\eta_{O W, j} \\ t O W, j \\ j=1,2, \ldots, M}} \text { Profit. }
$$

The profit equation is displayed in Eq. 2, where $E$ is calculated from Eq. 21 and $P C$ is found using production data. The demand is calculated by either external customer demand or by an internal standard if a certain level of inventory must be maintained. If the inserted energy opportunity window at machine $j$ is too large, i.e. 
$t_{O W, j}>d_{i}^{*}$, it will lead to the slowest machine idling from being starved/blocked, thus leading to permanent production loss. Therefore, the inserted opportunity window must be less than $d_{i}^{*}$. If the energy opportunity window is too small, more energy will be consumed while the machine warms up than energy saved from turning off machine $j$. This results in a greater energy cost, which leads to the lower bound of $t_{O W, j}$ :

$$
\begin{aligned}
t_{O W, j} P_{i d, j} & >E\left[t_{w, j}^{i}\right] P_{w, j} \\
t_{O W, j} & >\frac{\omega_{j} \beta_{j}}{\alpha_{j}} .
\end{aligned}
$$

Thus, the length of the inserted opportunity window is bounded by:

$$
\frac{\omega_{j} \beta_{j}}{\alpha_{j}}<t_{O W, j}<d_{i}^{*}, \quad j=1,2, \ldots, M, \quad i=1, \ldots, \eta_{O W, j} .
$$

This provides the bounds for $t_{O W, j}$, where the energy saved from the opportunity window must be larger than the expected energy consumed during the machine warm up period and must be less than the calculated opportunity window at that time. The control methodology builds upon the work in [30] by allowing the buffer levels to reach a certain threshold $\left(B_{0, j}\right)$ before taking the opportunity window and then turning the machine back on after $t_{O W, j}$. The pseudo code for the control algorithm is displayed below:

1. Begin shift

2. Collect pertinent data from buffer levels and machine states

3. If $b_{j}=B_{0, j}, \forall j<M^{*}$ turn off machine $j-1$ for $t_{O W, j-1}$.

(a) After $t_{O W, j-1}$ turn on machine $j-1$

4. If $b_{j} \neq B_{0, j}, \forall j<M^{*}$, go to step 5

5. If $b_{j}=B_{0, j}, \forall j>M^{*}$ turn off machine $j$ for $t_{O W, j}$.

(a) After $t_{O W, j}$ turn on machine $j$

6. If $b_{j} \neq B_{0, j}, \forall j>M^{*}$ return to step 2.

A flow chart of the control scheme is presented in Fig. 2. Each machine will run the control methodology as presented in Fig. 2 with a time step, ts. The machine will turn off when the buffer level reaches a specific threshold $\left(B_{0, j}\right)$ and then will turn on again after a specific amount of time $\left(t_{O W}\right)$. An example of the control methodology is illustrated in Fig. 3. The buffer threshold, $B_{0, j}$, depends on a specific scenario for each production line. Based on our simulation studies and experience, we normally determine the threshold to be approximately $95 \%$ of the buffer capacity [28]. In this 


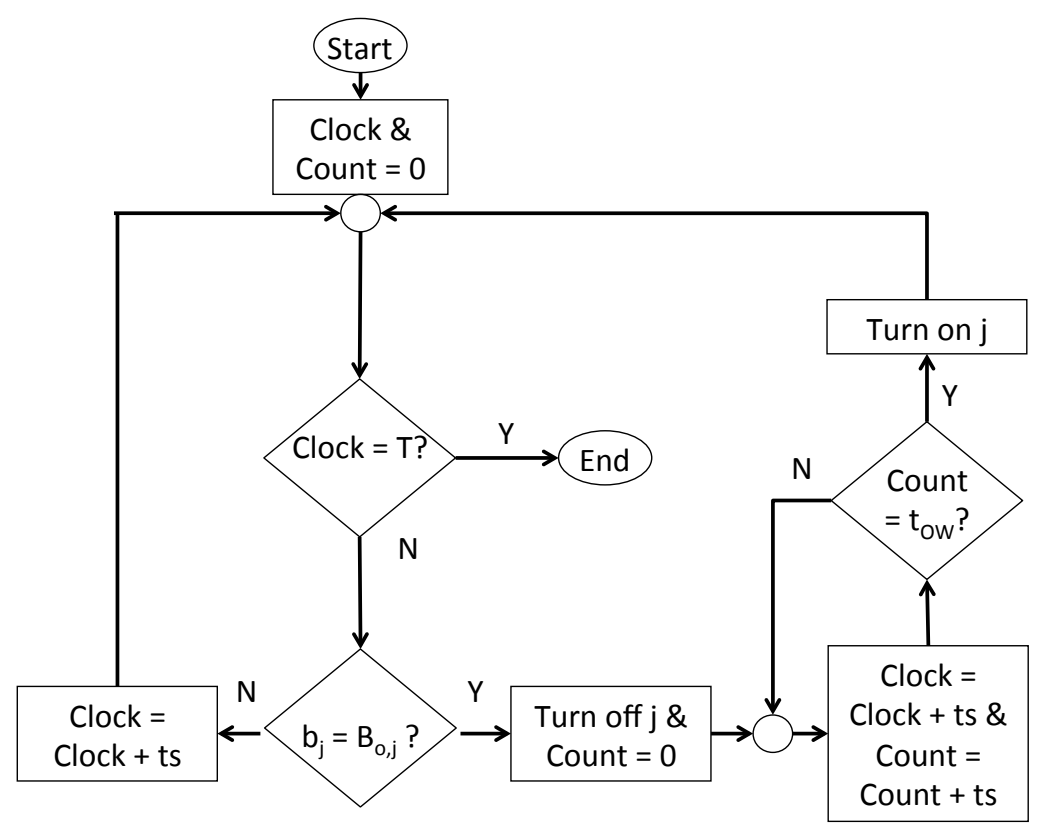

Figure 2: Control Methodology Flow Chart for machine $j$

specific example as shown in Fig. 3, the threshold $B_{0, j}$ is determined to be 50 parts, which is $95 \%$ of the buffer capacity. Once the buffer level reaches the threshold the machine is switched off for a period of 25 minutes and then it is switched back on. From our previous research, we found that the optimal $t_{O W, j}$ is approximately $70 \%$ of the upper bound [28]. This pattern is repeated until the end of the shift. This example also displays a random disruption event approximately 350 minutes into the shift. When the machine is switched on after the inserted opportunity window, it experiences a random failure, thus causing the buffer level to take longer to reach the 50 part threshold. By following this control scheme, the risk of production loss is lowered for the manufacturing line. The results are shown in the simulation case study. 


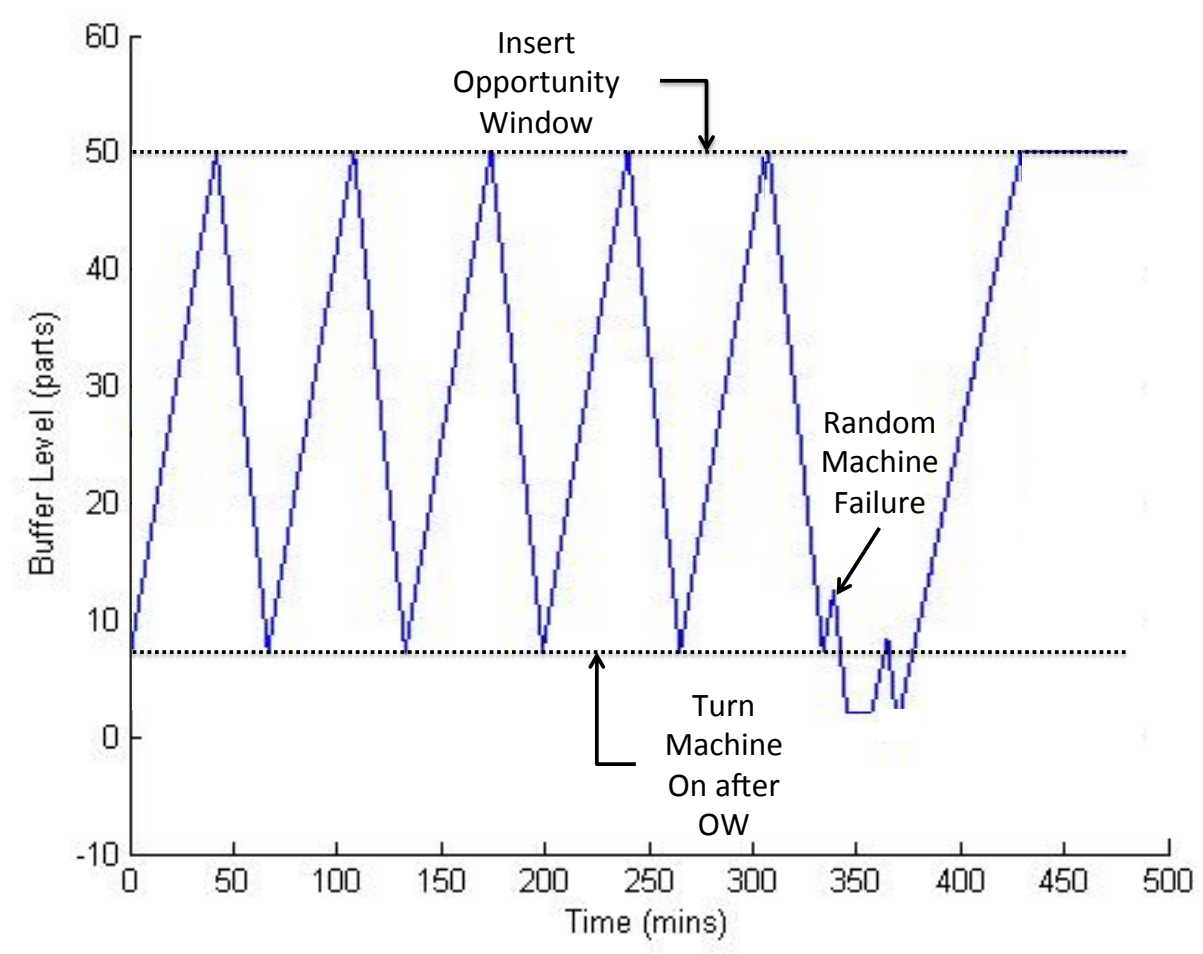

Figure 3: Control Methodology Buffer Thresholds

\section{SIMULATION CASE STUDYS}

Numerical simulation studies are performed to validate the energy economic methods presented previously. The return on investment strategy is tested in Section 5.1 while the control methodology is presented in Section 5.2.

\subsection{Economic Analysis Numerical Study}

A real automotive production line composed of 15 machines and 14 buffers is adopted for the case study to further demonstrate the return on investment analysis. The system parameters are recorded from the real production data and are mocked up for confidential reasons. The mean time to repair (MTTR) and the mean cycles between failure (MCBF) are assumed to be exponentially distributed based on this real data. The production parameters are shown in Table 1 . The idle power is assumed to be $70 \%\left(\alpha_{j}=0.7\right)$ of the power consumed during the production of parts and the warm up power is assumed to be $90 \%\left(\beta_{j}=0.9\right)$ of the power consumed during the production of parts for each machine. The mean time to warm up time after 
Table 1: Production Line Parameters: Case Study

\begin{tabular}{cccccccccccccccc}
\hline & $m_{1}$ & $m_{2}$ & $m_{3}$ & $m_{4}$ & $m_{5}$ & $m_{6}$ & $m_{7}$ & $m_{8}$ & $m_{9}$ & $m_{10}$ & $m_{11}$ & $m_{12}$ & $m_{13}$ & $m_{14}$ & $m_{15}$ \\
\hline $1 / \tau(\mathrm{p} / \mathrm{m})$ & 2.5 & 1.3 & 1.7 & 1.2 & 1.8 & 1.5 & 2.1 & 0.9 & 0.5 & 1.2 & 2.3 & 1.6 & 1.9 & 2.4 & 1.7 \\
MTTR (min) & 13 & 11 & 13 & 14 & 18 & 11 & 13 & 11 & 9 & 12 & 18 & 11 & 13 & 11 & 13 \\
MCBF (cyc) & 250 & 270 & 300 & 350 & 325 & 205 & 245 & 250 & 100 & 265 & 350 & 200 & 280 & 275 & 400 \\
Efficiency (\%) & 89 & 95 & 93 & 95 & 91 & 93 & 90 & 96 & 95 & 94 & 89 & 92 & 92 & 91 & 94 \\
$P_{p p}(\mathrm{~kW})$ & 25 & 30 & 21 & 25 & 40 & 10 & 43 & 40 & 17 & 45 & 28 & 25 & 15 & 21 & 30 \\
\hline \hline Buffer & - & $B_{2}$ & $B_{3}$ & $B_{4}$ & $B_{5}$ & $B_{6}$ & $B_{7}$ & $B_{8}$ & $B_{9}$ & $B_{10}$ & $B_{11}$ & $B_{12}$ & $B_{13}$ & $B_{14}$ & $B_{15}$ \\
Capacity & - & 20 & 30 & 15 & 20 & 25 & 20 & 25 & 35 & 30 & 25 & 30 & 25 & 20 & 15 \\
\hline
\end{tabular}

Table 2: Economic Analysis

\begin{tabular}{cccccccccccccccc}
\hline Option 1 & $m_{1}$ & $m_{2}$ & $m_{3}$ & $m_{4}$ & $m_{5}$ & $m_{6}$ & $m_{7}$ & $m_{8}$ & $m_{9}$ & $m_{10}$ & $m_{11}$ & $m_{12}$ & $m_{13}$ & $m_{14}$ & $m_{15}$ \\
\hline$\Delta P_{p p}(\mathrm{~kW})$ & 1 & 2 & 2 & 2 & 2 & 1 & 4 & 2 & 2 & 3 & 2 & 2 & 1 & 2 & 2 \\
$C T R(\$)$ & $1.2 \mathrm{k}$ & $2.2 \mathrm{k}$ & $2.1 \mathrm{k}$ & $3.6 \mathrm{k}$ & $2.1 \mathrm{k}$ & $1.1 \mathrm{k}$ & $4.1 \mathrm{k}$ & $2.9 \mathrm{k}$ & $4.6 \mathrm{k}$ & $4.9 \mathrm{k}$ & $2.4 \mathrm{k}$ & $2.2 \mathrm{k}$ & $1.1 \mathrm{k}$ & $2.2 \mathrm{k}$ & $2.3 \mathrm{k}$ \\
\hline \hline Option 2 & $m_{1}$ & $m_{2}$ & $m_{3}$ & $m_{4}$ & $m_{5}$ & $m_{6}$ & $m_{7}$ & $m_{8}$ & $m_{9}$ & $m_{10}$ & $m_{11}$ & $m_{12}$ & $m_{13}$ & $m_{14}$ & $m_{15}$ \\
\hline$\Delta P_{p p}(\mathrm{~kW})$ & 1 & 2 & 2 & 2 & 2 & 1 & 4 & 2 & 2 & 3 & 2 & 2 & 1 & 2 & 2 \\
$C T R(\$)$ & $1.5 \mathrm{k}$ & $2.5 \mathrm{k}$ & $2.4 \mathrm{k}$ & $2.7 \mathrm{k}$ & $2.2 \mathrm{k}$ & $1.4 \mathrm{k}$ & $4.2 \mathrm{k}$ & $2.2 \mathrm{k}$ & $2.2 \mathrm{k}$ & $3.3 \mathrm{k}$ & $2.3 \mathrm{k}$ & $2.4 \mathrm{k}$ & $1.2 \mathrm{k}$ & $2.5 \mathrm{k}$ & $2.6 \mathrm{k}$ \\
\hline \hline Option 3 & $m_{1}$ & $m_{2}$ & $m_{3}$ & $m_{4}$ & $m_{5}$ & $m_{6}$ & $m_{7}$ & $m_{8}$ & $m_{9}$ & $m_{10}$ & $m_{11}$ & $m_{12}$ & $m_{13}$ & $m_{14}$ & $m_{15}$ \\
\hline$\Delta P_{p p}(\mathrm{~kW})$ & 7 & 10 & 5 & 7 & 10 & 2 & 12 & 9 & 5 & 11 & 5 & 7 & 4 & 3 & 8 \\
$C T R(\$)$ & $23 \mathrm{k}$ & $35 \mathrm{k}$ & $21 \mathrm{k}$ & $37 \mathrm{k}$ & $23 \mathrm{k}$ & $37 \mathrm{k}$ & $49 \mathrm{k}$ & $34 \mathrm{k}$ & $20 \mathrm{k}$ & $60 \mathrm{k}$ & $23 \mathrm{k}$ & $36 \mathrm{k}$ & $25 \mathrm{k}$ & $28 \mathrm{k}$ & $35 \mathrm{k}$ \\
\hline
\end{tabular}

the $i^{\text {th }}$ downtime event is 1 minute $\left(\omega_{j}=1 \mathrm{~min}\right)$. The cost of energy is $\$ 0.14 / k W h$ and the profit per part is $\$ 50$ per part. To test the return on investment strategy, three options are presented: option 1 is the scenario where a machine part is replaced with a more energy efficient version during a regular production shift, option 2 is the case where the same part is replaced, but during an overtime shift when there is no production, and option 3 is the situation when the entire machine is replaced with a more energy efficient version. We assume that $C_{R e g, j}$ is $\$ 70$ per hour and $C_{O T, j}$ is $\$ 140$ per hour for each machine. We also manage the replacement of the part during a regular shift when the machine has its maximum opportunity window therefore limiting production loss. The parameters for each option are shown in Table 2. The $C T R$ is in thousands of dollar, i.e. $5 \mathrm{k}=\$ 5,000$. We utilize Eq. 20 to calculate the expected number of days the investment will break even and then compare that with the simulation break even point for the investment. The results are shown in Fig. 4-6. The diamonds represent the theoretical break even point calculated with Eq. 20. The squares are the break even points calculated from the simulation model for each option. The results illustrate that the simulation break even point is within $10 \%$ error (as shown with the error bars) for each option and each machine. The 


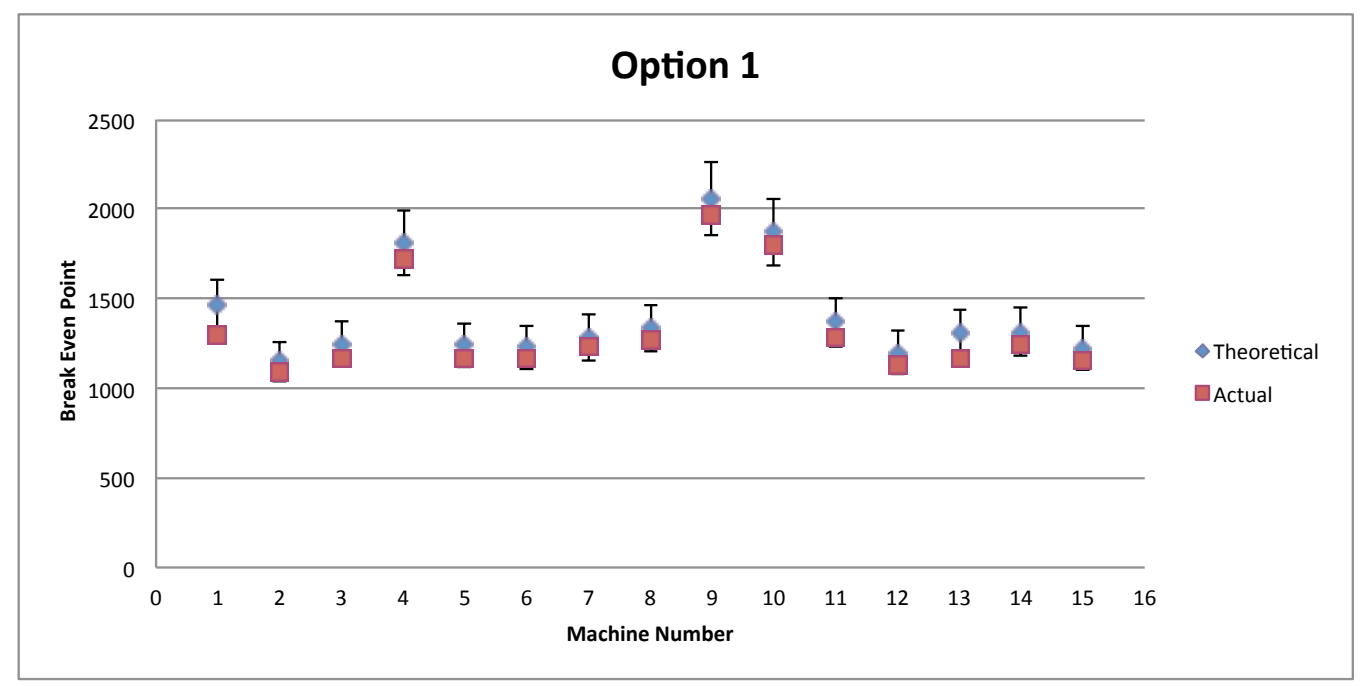

Figure 4: BREAK EVEN RESULTS - OPTION 1

best option is replacing a part during an overtime shift (option 2) for machine 9, which leads to an expected break even point of 997 days, while the simulation study calculates 951 days. The next section will use this return on investment analysis in conjunction with the opportunity window control scheme to maximize profits for the production line.

\subsection{Opportunity Window Control Numerical Study}

This study uses the same production line as seen in Table 1. The return on investment strategy is utilized in conjunction with the control methodology from Section 4. This is labeled as "OW Control." The cost of energy is still $\$ 0.14 / k W h$ and the profit per part is still $\$ 50$ per part. The back log cost, $c_{b}$, is $\$ 15$ per part and the inventory cost, $c_{i}$, is $\$ 5$ per part. The production line is run for a 5 year time period, assuming one 8 hour shift per day, with no opportunity window control methodology and no machines or parts replaced with upgraded versions. The profit for a 5 year period is calculated and recorded as the baseline scenario. One part in machine 9 is then replaced based on the break even and return on investment analysis as shown in Table 2. The opportunity window control methodology is then used and the line is simulated for another 5 year period with a part from machine 9 replaced with a more energy efficient version with a $2 \mathrm{~kW}$ reduced power consumption. The results are recorded and compared with the baseline scenario and three other control scenarios. The various scenarios are presented in Table 3. The baseline scenario has no control methodology and no replacement strategy. Control 1-3 have 


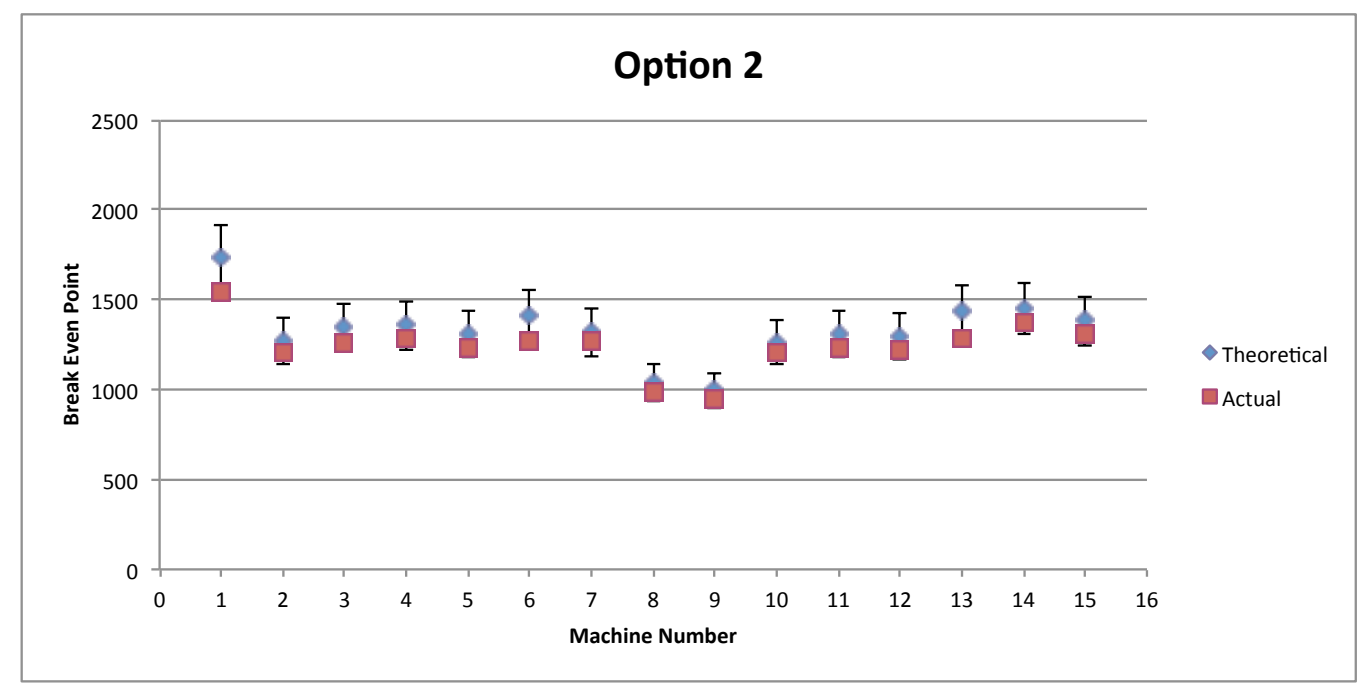

Figure 5: BREAK EVEN RESULTS - OPTION 2

Table 3: Control Strategies

\begin{tabular}{cccccc}
\hline- & Baseline & Control 1 & Control 2 & Control 3 & OW Control \\
\hline Control & None & Set & Set & Set & OW Control \\
Replacement & None & None & Cheapest & Power Decrease & ROI \\
\hline
\end{tabular}

set maintenance schedules, where each machine is turned off for a set period of time based on a pre made schedule. This is a typical control methodology in industry. In Control 1 no machine or part is replaced. Control 2 replaces a part based on the cheapest investment, which replaces a part in machine 6 during a regular shift (option 1). Control 3 replaces the entire machine (option 3) based on the largest decrease in power consumption, which replaces machine 10. Lastly, the OW Control is the scenario where the control from Section 4 is utilized in conjunction with the return on investment strategy. Figure 7 displays the production count of the baseline scenario versus the control scenarios. The demand is 230 parts per day, which is 83,950 parts per year, which is met by all of the control scenarios and the baseline situation. The baseline scenario produces 18,250 parts over the demand of the facility, while the OW control scenario produces 16,263 parts more than the demand. In the "OW Control" scenario, by reducing the number of parts produced past the demand threshold in the control scenario and replacing a part within machine 9 , the overall energy consumption decreases by 1,519,404 $k W h$. Thus, running the opportunity window control scheme while using the return on investment strategy leads to a decreased energy consumption with no impact to the production demand. This 


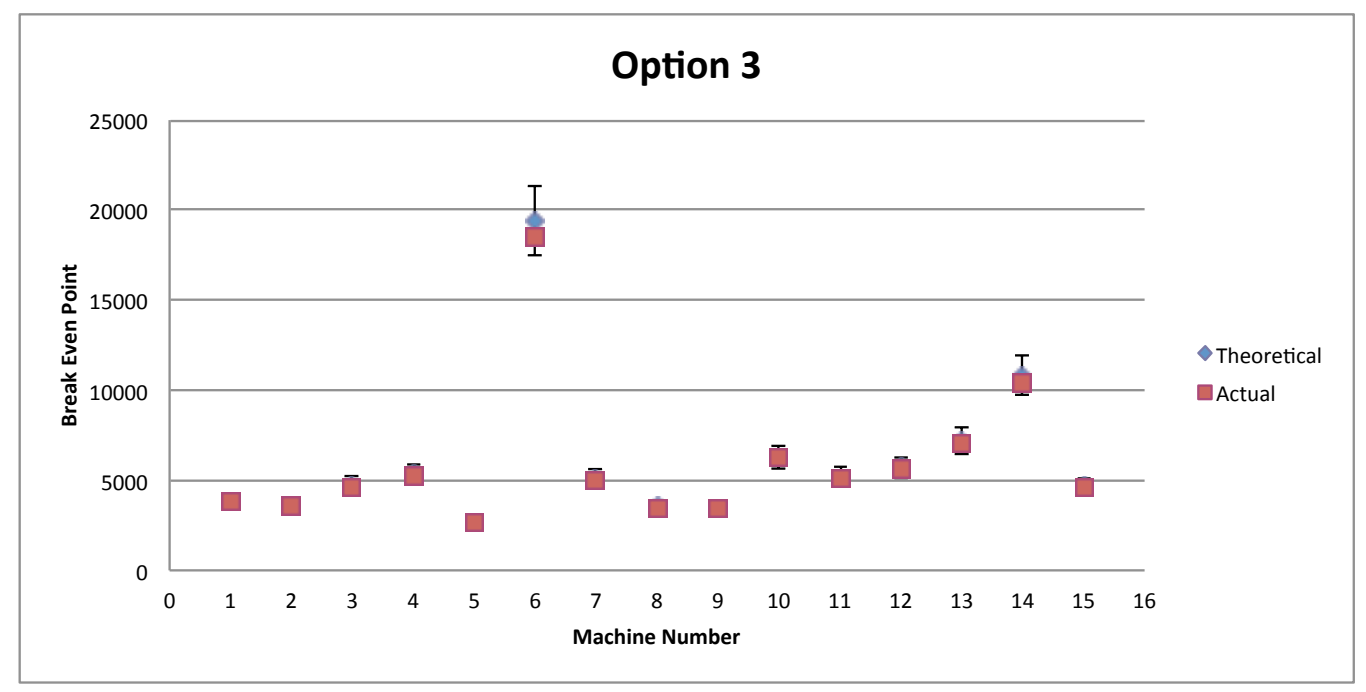

Figure 6: BREAK EVEN RESULTS - OPTION 3

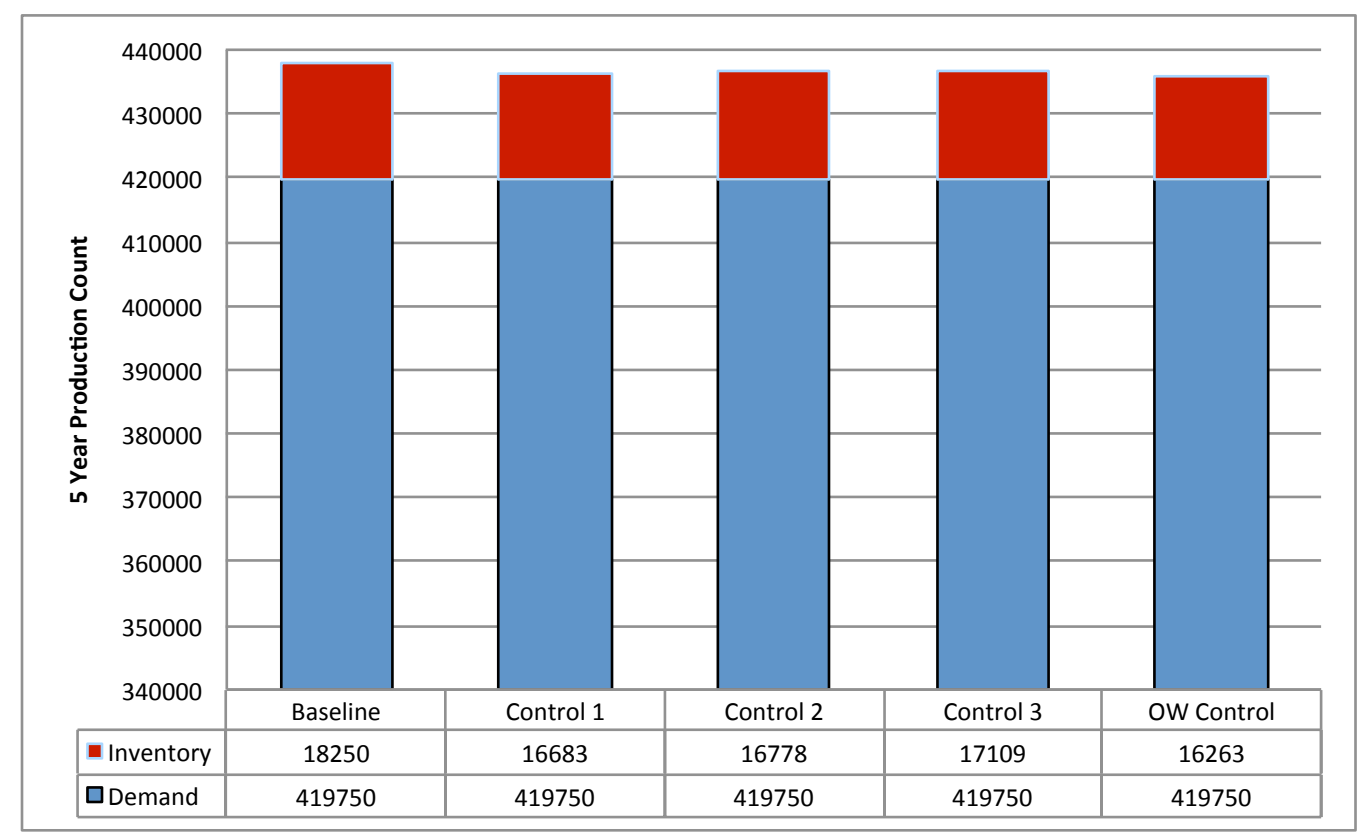

Figure 7: 5 YEAR PRODUCTION COUNT

leads to an overall profit increase of $\$ 121,107.36$ over five years from the baseline and $\$ 19,555.49$ from Control 2 as displayed in Fig. 8. 


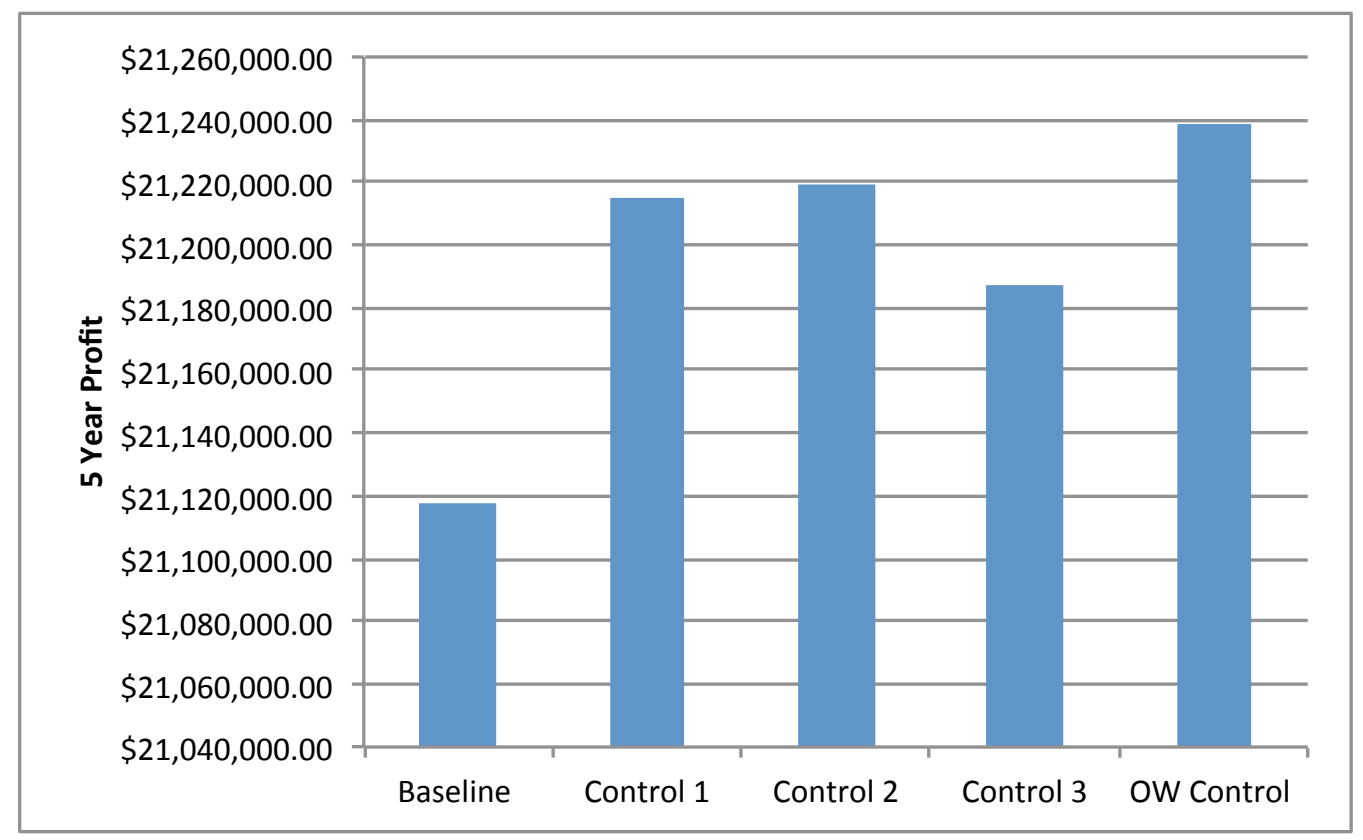

Figure 8: 5 YEAR PROFIT

\section{CONCLUSIONS \& FUTURE WORK}

This paper investigates the energy economics of a manufacturing facility by creating a return on investment strategy for replacing machines or parts with more energy efficient versions. The profit of the production plant is explored to include both production and energy costs. The energy dynamics of the production system are studied and incorporated into an energy economic analysis. The analysis provides the plant manager with the quantitative tools to increase profit in the long term by replacing the machine or part of a machine, which will lead to the largest return on investment. A control methodology is introduced that utilizes inserted opportunity windows to reduce overall energy consumption with minimal throughput impact. A case study is studied to validate the opportunity window control scheme in conjunction with the energy economic method. Future work will expand the energy economic analysis to include replacing a machine with a more production efficient version (i.e. MCBF is reduced). This will provide the plant manager with another tool to increase the overall efficiency of the manufacturing facility. An optimal control methodology will also be developed to further increase profits by decreasing energy consumption while increasing production output. 


\section{ACKNOWLEDGEMENTS}

This research was funded by the National Science Foundation under CAREER AWARD CMMI-1351160.

[1] U.S. Energy Information Administration. (2014). Energy Consumption by Sector and Source http://www.eia.gov/forecasts/aeo/pdf/tbla2.pdf

[2] U.S. Energy Information Administration. (2014). Consumption E6 Efficiency http://www.eia.gov/consumption/

[3] U.S. Energy Information Administration. (2014). Manufacturing Energy Consumption Survey http://www.eia.gov/consumption/manufacturing/

[4] Guerrero, C.A., Wang, J., Li, J., Arinez, J., Biller, S, Huang, N., and Xiao, G. (2011). "Production system design to achieve energy savings in an automotive paint shop, " International Journal of Production Research, 49(22): 6769-6785, DOI: $10.1080 / 00207543.2010 .535042$.

[5] U.S. Environmental Protection Agency (EPA). (2009). "Chapter 3: Energy Assessment Strategies", Lean and Energy Toolkit.

[6] Bohringer, C., Moslener, U., Oberndorfer, U., and Ziegler, A., (2012.) "Clean and productive? Empirical evidence from the German manufacturing industry," Research Policy, 41(2): 442-451, DOI: 10.1016/j.respol.2011.10.004.

[7] Mann, L.J., (1983), Maintenance Management, Lexington Books, Lexington, MA.

[8] Levitt, J., (1997), The Handbook of Maintenance Management, Industrial Press, NY.

[9] Yang, Z. ,Chang, Q., Djurdanjovic, D., Ni., J, and Lee, J. (2006). "Maintenance Priority Assignment Utilizing On-line Production Information," ASME Transaction, Journal of Manufacturing Science and Engineering, 129(2), pp. 435-446. DOI:10.1115/1.2336257.

[10] Dekker, R., (1995), "Integrating Optimization, Priority Setting, Planning, and Combining of Maintenance Activities," European Jouranl of Operational Research, 72, pp. 225-240. 
[11] Shen, Q.P., Lo, K.K., and Wang, Q., (1998), "Priority Setting in Maintenance Management: A Modified Multi-Attribute Approach Using Analytic Hierarchy Process," Construction Management and Economics 16, pp. 693-702.

[12] Haapala, K.R., Zhao, F., Camelio, J., Sutherland, J.W., Skerlos, S.J., Dornfeld, D.A., Jawahir, I.S., Clarens, A.F., and Rickli, J.L. (2013). "A Review of Engineering Research in Sustainable Manufacturing" ASME Transaction, Journal of Manufacturing Science and Engineering 135(4), DOI: 10.1115/1.4024040.

[13] Singh, S., Olugu, E.U., and Fallahpour, A. (2014). "Fuzzy-based sustainable manufacturing assessment model for SMEs, "Clean Technologies and Envinronmental Policy, 16(5): 847-860. DOI: 10.1007/s10098-013-0676-5.

[14] Brundage, M., Chang, Q., Li, Y., Arinez, J, and Xiao, G. (2014). "Sustainable Manufacturing Performance Indicators for a Serial Production Line," IEEE Transactions on Automation Science and Engineering, Vol. PP, No. 99, pp 1-12.

[15] Despeisse, M., Ball, P.D., and Evans, S. (2012). "Modelling and Tactics for Sustainable Manufacturing: An Improvement Methodology," Sustainable Manufacturing, pp. 9-16. DOI: 10.1007/978-3-642-27290-5_2.

[16] Bunse, K., Vodicka, M., Schonsleben, P., Brulhart, M., and Ernst, F.O., (2011). "Integrating energy efficiency performance in production management - gap analysis between industrial needs and scientific literature," Journal of Cleaner Production, 19(6-7): 667-679. DOI: 10.1016/j.jclepro.2010.11.011.

[17] Decanio, S.J. and Watkins, W.E. (1998.) "Investment in energy efficiency: do the characteristics of firms matter?", The Review of Economics and Statistics 80:95-107.

[18] Gellings, C.W. , (1985). "The concept of demand-side management for electric utilities", Proceedings of the IEEE, 73(10): 1468-1470. DOI: 10.1109/PROC.1985.13318.

[19] Brundage, M., Chang, Q., Li, Y., Arinez, J, and Xiao, G. (2013). "Energy efficiency management of an integrated serial production line and HVAC system," IEEE Transaction on Automation Science and Engineering, Vol. 11, No. 3, pp 789-797. DOI: 10.1109/TASE.2013.2284915.

[20] Sevast'yanov, B.A., (1962). "Influence of Storage Bin Capacity on the Average Standstill Time of a Production Line," Theory of Probability and its Applications, 7(4):429-438., 1962. 
[21] Wijngaard, J., (1979). "The Effect of Interstage Buffer Storage on Output of Two Unreliable Production Units in Series with Different Production Rates," AIIE Transactions, 11(1):42-47.

[22] Gershwin S. and Schick, I., (1980). "Continuous Model of an Unreliable TwoStage Material Flow System with a Finite Inter-stage Buffer," Technical Report 1039, Massachusetts Institute of Technology, MA.

[23] Gershwin, S., (1994). Manufacturing Systems Engineering, Prentice-Hall, Englewood Cliffs, NJ.

[24] Li, J., Meerkov J., Production Systems Engineering, Springer, 2008.

[25] Chang, Q., Xiao, G., Biller, S., and Li, L., (2012). "Energy Saving Opportunity Analysis of Automotive Serial Production Systems," IEEE Transaction on Automation Science and Engineering. DOI: 10.1109/TASE.2012.2210874.

[26] Chang, Q., Biller, S., Xiao, G., and Liu, J., (2010). "Transient Analysis of Downtimes and Bottleneck Dynamics in Serial Manufacturing Systems," ASME Transaction, Journal of Manufacturing Science and Engineering, 132(5), 051015.

[27] Liu, J., Chang, Q., Xiao, G., and Biller, S., (2012). "The Costs of Downtime Incidents in Serial Multi-Stage Manufacturing Systems," ASME Transaction, Journal of Manufacturing Science and Engineering, 134(2) 02101.

[28] Brundage, M., Chang, Q., Li, Y., Arinez, J., and Xiao, G., (2015) "Implementing a Real-Time, Energy Efficient Control Methodology to Maximize Manufacturing Profits", IEEE Transactions on Systems, Man and Cybernetics: Systems. Accepted.

[29] Investopedia (2014). Return on Investment (ROI) http://www.investopedia.com/terms/r/returnoninvestment.asp

[30] Chang, Q., Ni., J, Bandyopadhyay, P., Biller, S., and Xiao, G., (2007). "Maintenance Opportunity Planning System," ASME Transaction, Journal of Manufacturing Science and Engineering, 129(3), pp. 661-668. DOI:10.1115/1.2716713. 